\title{
Preliminary investigation into the effects of SlimBiome on food cravings and responsiveness to the food environment in healthy overweight and obese women following standard nutritional advice
}

\author{
I. Achebe ${ }^{1}$, H. Brennan ${ }^{1}$, S. Kolida ${ }^{2}$, S. OHara ${ }^{2}$, H. Lightowler ${ }^{1}$ and L. Ahlstrom ${ }^{1}$ \\ ${ }^{1}$ Oxford Brookes Centre for Nutrition and Health, Oxford Brookes University, Oxford, OX3 OBP, UK and ${ }^{2}$ OptiBiotix \\ Health Plc, Heslington, York, YO10 5DG, UK.
}

The prevalence of obesity is rising, with current UK statistics indicating that $68 \%$ of men and $58 \%$ of women are classified as overweight or obese ${ }^{(1)}$. Consequently, effective weight management is of increasing importance and considerable attention is being placed on the use of functional ingredients in foods that are less energy dense and more satiating. The aim of this study was to investigate the effect of SlimBiome, a commercially available product, on food cravings and the responsiveness of individuals to the food environment, which are characteristics associated with hunger ${ }^{(2,3)}$, in overweight and obese women following standard nutritional advice. SlimBiome contains fibre (glucomannan), prebiotics and chromium, which have been shown to increase satiety and reduce energy intake, hunger and fat cravings in overweight and obese adults ${ }^{(4-6)}$.

A double-blind, randomised, placebo-controlled study was conducted in 20 overweight and obese women over a 4-week period. Inclusion criteria included women aged 18-65 years, with a body mass index between $25-35 \mathrm{~kg} / \mathrm{m}^{2}$, no significant health problems, and no history of dieting within the month prior to starting the study. Participants attended three study visits at week 0 , week 1 and week 4 . At week 0 they were randomly assigned to receive the test drink or placebo and were instructed to consume the drink three times a day for 4 weeks. At each visit, participants rated subjective sensations of appetite before and after consuming the test drink or placebo and completed the Control of Eating Questionnaire (CofEQ) ${ }^{(7)}$ and the Power of Food Scale (POF) ${ }^{(8)}$. During the week 0 visit, standard nutritional advice based on the Eat Well Guide was provided to all participants. Differences in week 0 and week 4 POF Scale scores for both groups were assessed using Wilcoxon signed-rank tests. Differences between group week 4 CofEQ scores were assessed using analysis of covariance (ANCOVA), including baseline scores from week 0 as covariates.

For the CofEQ, ratings in response to 'How hungry have you felt?' at week 4 were significantly lower in the treatment group compared to the placebo group $(P=0.034)$. No significant differences were observed between the groups at week 4 for CofEQ items assessing frequency or strength of food cravings $(P>0.05$ for all items). For the POF Scale, scores decreased significantly after the 4-week intervention period in both the placebo group $(P=0.035)$ and the treatment group $(P=0.007)$.

In conclusion, subjective feelings of hunger measured by the CofEQ were significantly reduced in women who consumed SlimBiome compared to placebo. Although cravings appeared to subside more in women consuming SlimBiome, no significant difference was seen compared to placebo. This may be due to both groups becoming less responsive to the food environment, a characteristic shown to predict strength of and ability to resist cravings ${ }^{(3)}$.

1. Department of National Statistics. Available from: https:/www.gov.uk/government/statistics/statistics-on-obesity-physical-activity-and-diet-england-2017.

2. Steel D, Kemps E \& Tiggemann M (2006 Jan 1) Appetite 46(1), 36-40.

3. Forman EM, Hoffman KL, McGrath KB et al. (2007 Oct 1) Behav Res Ther 45(10), 2372-86.

4. Parnell JA \& Reimer RA (2009 Apr 22) Am J Clin Nutr 89(6), 1751-9.

5. Archer BJ, Johnson SK, Devereux HM \& Baxter AL (2004 Apr) Br J Nutr 91(4), 591-9.

6. Anton SD, Morrison CD, Cefalu WT et al. (2008 Oct 1) Diabetes Technol Ther 10(5), 405-12.

7. Dalton M, Finlayson G, Hill A \& Blundell J (2015 Dec) Eur J Clin Nutr 69(12), 1313.

8. Cappelleri JC, Bushmakin AG, Gerber RA et al. (2009 Aug) Int J Obes 33(8), 913. 